\title{
Diagnosis of Pulpitis from Dental Panoramic Radiograph Using Histogram of Gradients with Discrete Wavelet Transform and Multilevel Neural Network Techniques
}

\author{
Antony Vigil ${ }^{*}$, Subbiah Bharathi ${ }^{2}$ \\ ${ }^{1}$ Department of Computer Science and Engineering, SRM Institute of Science and Technology, Ramapuram Campus, Chennai \\ 600089 , India \\ ${ }^{2}$ Department of Electronics and Communication Engineering, SRM Institute of Science and Technology, Ramapuram Campus, \\ Chennai 600089, India
}

Corresponding Author Email: antonym@srmist.edu.in

https://doi.org/10.18280/ts.380532

Received: 16 July 2021

Accepted: 20 September 2021

\section{Keywords:}

dental panoramic radiograph, modified $k$ means, pulpitis, discrete wavelet transform, multi-level neural network

\begin{abstract}
Radiograph plays the major role of diagnosis, treatment and surgery in the Dental field. There are many types of Intra and extra oral radiographs in which Dental Panoramic Radiograph helps in visualising the full view of the oral cavity. Pulpitis is the dental diseases caused due to the inflammation of the dental pulp from untreated caries, trauma or multiple restorations which leads to Apical Periodontitis. To predict the severity of pulp vitality pulp inflammation has to be evaluated. Radiographs helps the dentist in diagnosing the extent of tooth decay and inflammation. An automatic diagnostic model is proposed using robust algorithms to diagnose pulpits. Dental Panoramic Radiograph is used in the proposed research to diagnose the pulpitis and to classify the normal teeth from the pulpitis. The collected images are pre-processed using Histogram Equalization and filtered using Gaussian and Median filters. Modified K-Means algorithm is applied to segment the bony and teeth area from the oral cavity area. Integral Histogram of Gradients with Discrete Wavelet Transform feature extraction techniques and Multi-Layer Neural Network Classifier is proposed to achieve the accuracy of $91.09 \%$ which can be used as an assistive tool by the dentist to diagnose pulpitis.
\end{abstract}

\section{INTRODUCTION}

Medical imaging plays the major role in the field of dentistry in diagnosis, treatment and surgery. For adult radiographs are used to detect decay between teeth, decay beneath the fillings, bone and root canal infection, tooth implants, bone loss, Pulpitis, cyst and tumours. For children radiographs helps to identify whether enough space is available in oral cavity to fit all teeth, to check the development of wisdom teeth and status of impacted teeth. Dental Radiographs can be classified as Intraoral and Extraoral Radiographs [1]. In Intraoral Radiographs X-ray films are placed inside the mouth whereas in Extraoral Radiographs X-ray films are placed outside the mouth. Bitewing, Periapical and Occlusal X-rays are Intraoral Radiographs. Panoramic, Tomograms and Cephalometric Projections are Extraoral Radiographs [2].

Dentists diagnose the severity of dental diseases like Dental caries, Tooth decay [3], periodontitis, cracked/broken teeth, pulpitis, Oral cancer using Dental radiographs. The Dental Pulp tissue inflammation is known as pulpitis which leads to Apical Periodontitis. Dental pulp comprises blood vessels, nerves and connective tissues within the tooth which provides nutrients and blood to the tooth [4]. Secondary development of tooth decay or caries is known as pulpitis which is caused by the infection of oral microorganisms.

Symptoms of pulpitis are continuous tooth pain, sensitivity in exposure to heat or cold, sensitivity or pain in the oral cavity, swelling in the face and fever. Pulpitis is caused by the bacterial infection that penetrates through the dentin and enamel to reach the pulp [5]. Severity of pulpitis results to root canal treatment and if not treated spreads to sinus, jaw and brain. Pulpitis can be diagnosed by dentist using pulp sensibility test. It is of three types like thermal test which is cold or heat test, Electrical pulp test which determines the pulp and pulp related tissues health. Dentist use radiographs to diagnose the severity of pulp inflammation. American Association of Endodontists [6] classifies the pulpal diseases in which two main types are Reversible and Irreversible pulpitis. Reversible pulpitis cannot be diagnosed using radiograph and it can be curable by removal of damage and placement of restoration. Irreversible pulpitis can be diagnosed using radiograph and in this stage the pulp cannot be recovered from its damage which results to death of pulp tissue. However, the tooth can be treated by removing the pulp or root canal treatment.

Talabani et al., evaluated the pulpal and periradicular tissue using clinical and radiographic methods for the patient's undergone endodontic treatment. Pulpitis are categorized as reversible and irreversible types and identified irreversible pulpitis is the main cause of root canal treatment [7].

Abella et al. evaluated the irreversible pulpitis periapical status using CBCT scanning and periapical radiographs. Periapical radiographs are used in the treatment of endodontic and also to access the treatment outcome. The result shows that the accuracy of CBCT was significantly higher than that of periapical radiograph [8].

Saidi et al. done a study in evaluating the accuracy of CBCT 
and Periapical radiography of endodontically treated teeth. The research was done five years after the endodontic treatment and found that $\mathrm{CBCT}$ is more reliable in detecting periapical lesions compared to radiographs [9].

Dental Panoramic Radiographs is an extra oral radiograph which helps to view the full view of the mouth. An automatic assistive model is proposed to diagnose the pulpitis dental disease by reducing dentists workload and human error [10]. Radiographs fails to disclose the features and anatomical structure of oral cavity due to the variation in intensity and technical difficulties while capturing the image. The captured image has to be processed using image processing algorithms like pre-processing, segmentation, feature extraction and classification using robust algorithms. Proper evaluation of pulp inflammation before treatment is the key to improve pulp vital [11]. The developed model can assist dentist in diagnosing Pulpitis and helps for further treatment.

\section{MATERIALS AND METHODS}

Dental Panoramic Radiograph visualize the entire structure of the teeth and the related structures. It is used to examine tumours, cysts, pulpitis, location and position of impacted teeth, fractures, grown pattern of jaws etc. Pulp is the internal part of the tooth that contains nutrition and blood vessels for the connective tissues. Clinical diagnosis indicates pulp as normal pulp, reversible pulpitis and irreversible pulpitis. Diagnosing the indications of pulp is not accurate if it's done only with clinical proof. So it would be better if radiographic diagnosis is done. However radiographic diagnosis of small caries and early stage of pulpitis would not be able to identified using panoramic radiograph.
Radiographic results are not prominent is diagnosing the dental diseases. Image processing techniques has to be applied to the input image which helps the researchers to develop the automatic model more efficiently. Edge detection techniques have been applied to calculate the number of pixels and Gaussian filter helps in smoothing the image to detect the healthy and decayed tooth.

Dental Panoramic Radiographs are used in the proposed research of diagnosing the pulpitis since the full view of the mouth with the anatomical structure of Molar, Premolar, Canine and Incisor can be visualized. Dimension and structure of each tooth varies from person to person and it's difficult to diagnose the dental diseases from the radiograph without processing the image. Robust computing algorithms like Media filter, modified K-Means, Integrated Histogram of Gradients (HoG), Discrete Wavelet Transform (DWT) and Multilayer Neural Network (MLNN) are proposed in diagnosing the pulpitis.

\subsection{Image acquisition}

Dentist use various Extraoral and Intraoral radiographs to diagnose the dental diseases based on their requirement. Totally 200 Dental Panoramic Radiographs are used in the research which was collected from various patients of age 3050 from SRM Dental College, Chennai. The anatomical structure of each tooth differs for each person. The images are captured with $70 \mathrm{KV}$ to $80 \mathrm{KV}$ voltage, $8 \mathrm{~mA}$ to $10 \mathrm{~mA}$ current and the size of the captured images are $1480 \times 2658$. The captured images are manually grouped as pulpitis and normal by the Dentist and the pulpitis tooth is labelled by Dentist which helps the researchers to train and validate the model. Figure 1 shows the block diagram for the proposed model in diagnosing pulpitis from DPR.

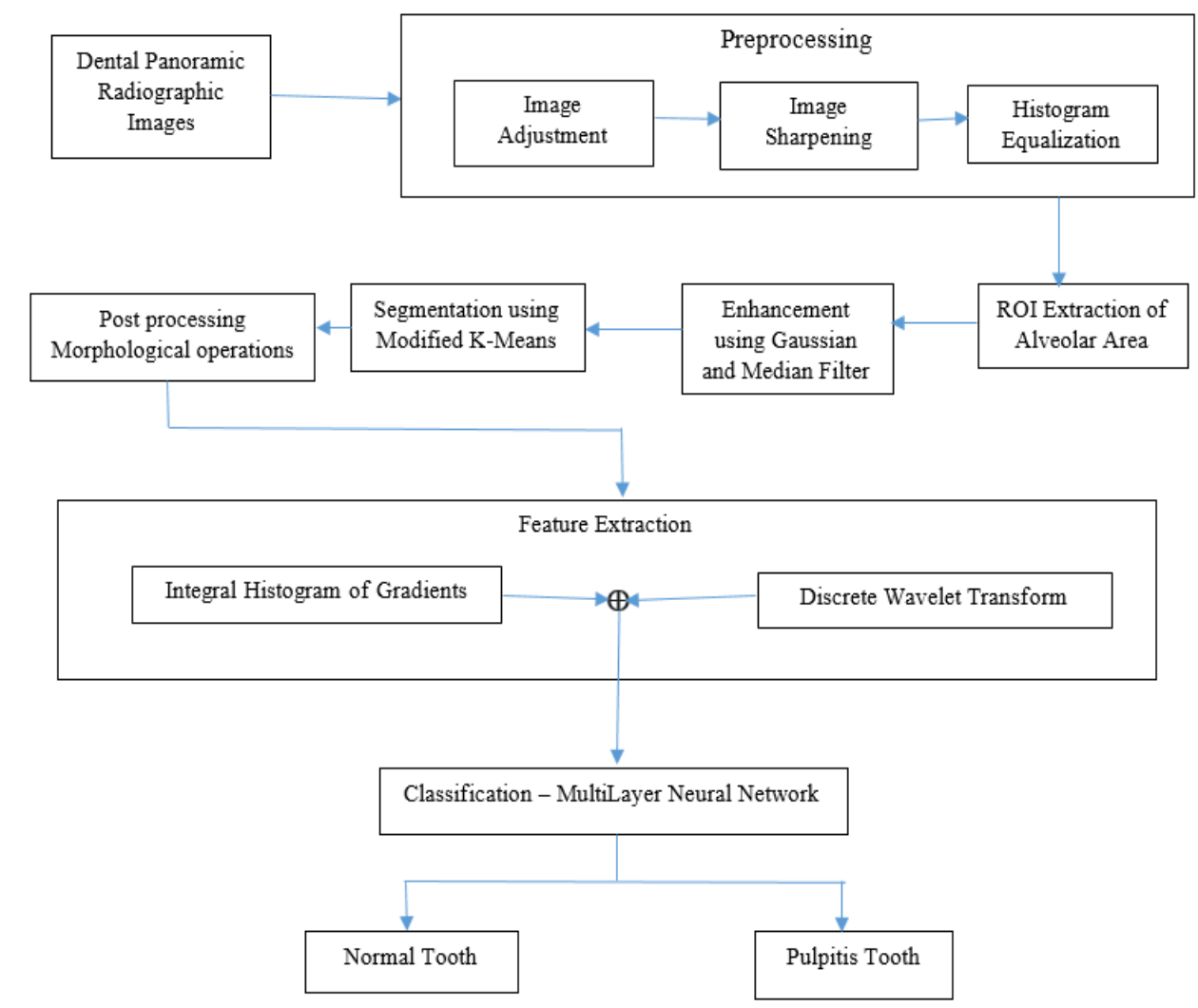

Figure 1. Proposed block diagram for diagnosis of pulpitis from dental panoramic radiograph 


\subsection{Image preprocessing}

The collected images have to be preprocessed initially for further processing due to the poor quality with blurred edges [12], variation in illumination, intensity orientation and capturing position of the images [13]. The captured images are adjusted, sharpened and then undergone Histogram Equalization to make the boundaries of teeth and bone more informative [14].

The proposed research is carried out in the teeth of Mandibular region and for better processing region of interest has to be extracted [15]. Alveolar area of mandibular region is considered as $\mathrm{ROI}$ and it is extracted by fixing the specified height and width dimensions.

\subsection{Enhancement using Gaussian and median filter}

Gaussian filter is a linear filter which reduces the noise of the input image. This filter uses a Gaussian function which defines the probability distribution of the noise or unwanted data [16]. While using the Gaussian filter to an image it has to be used as two dimensional Gaussian function which is a product of two 1D Gaussian functions which can be given as below.

$$
\mathrm{G}(\mathrm{x}, \mathrm{y})=\left(1 / 2 \pi \sigma^{2}\right) \mathrm{e}^{(\mathrm{x} 2+\mathrm{y} 2) / 2 \sigma 2}
$$

where, $\mathrm{x}$ and $\mathrm{y}$ are the horizontal and vertical axis distances from the origin.

Median filter is used to smooth the input blurred image [17] and to remove noise [18]. Since the low frequency values are subtracted, the high frequency values are left out with the original image which sharpens the boundaries and enhanced it.

Let $S_{\mathrm{ij}}$ represents the set of coordinates for the window of size $\mathrm{p} \mathrm{x} \mathrm{q}$ and the centre point is $(\mathrm{i}, \mathrm{j})$. The input image median value in the region defined by $S_{i j}$ is computed by the Median filter. The output image $f^{\prime}(i, j)$ is given by,

$$
\mathrm{f}^{\prime}(\mathrm{i}, \mathrm{j})=\underset{(\mathrm{s}, \mathrm{t}) \in \mathrm{S}_{\mathrm{ij}}}{\operatorname{median}}\{\mathrm{g}(\mathrm{s}, \mathrm{t})\}
$$

where, $g(s, t)$ is degraded image which can be obtained by the combination of input image $f(x, y)$ and noise $n(x, y)$.

\subsection{Segmentation using modified K-means}

Segmentation is the main process which partitions the various parts of image into segments [19]. In our research work the bone and tooth region has to be segmented from the other parts of oral cavity. Modified K-Means algorithm is proposed which uses centroid of K clusters [20]. The proposed Modified K-means Algorithm is shown in Algorithm 1.

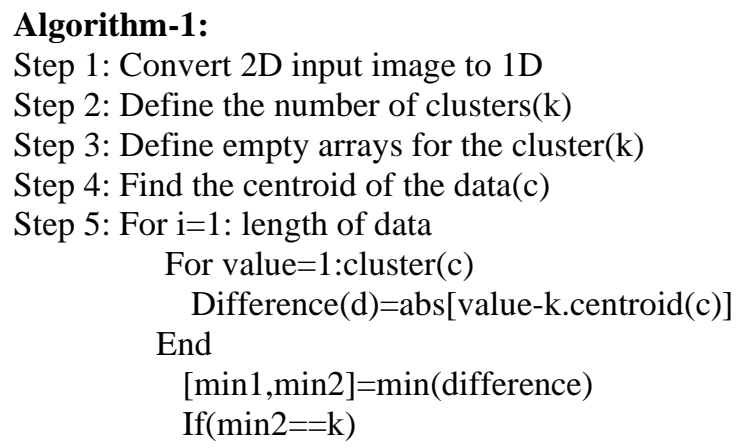

$$
\begin{aligned}
& \text { Cluster }(\mathrm{i})=\text { value } \\
& \text { End }
\end{aligned}
$$

Step 6: Reshape each cluster(k)

Step 7: Display the output image

The proposed segmentation algorithm clusters the teeth and bone area which supports the researchers for further processing.

\subsection{Post processing morphological operations}

Morphological operations are applied to the images that is processed based on structures and shapes. Erosion and Open Morphological operations are used in the work to split the alveolar area label from the occlusion. Erosion operator takes the structuring element and image as input and thins or shrinks the object. Consider $\mathrm{P}$ and $\mathrm{Q}$ as structuring elements. Then erosion of $\mathrm{P}$ and $\mathrm{Q}$ can be defined as:

$$
\mathrm{P} \ominus \mathrm{Q}=\{\mathrm{x} \mid(\mathrm{Q}) \mathrm{x} \subseteq \mathrm{P}\}
$$

which says that the erosion of $\mathrm{P}$ by $\mathrm{Q}$ is set of all points in $\mathrm{x}$ such that $\mathrm{x}$ translation in $\mathrm{Q}$ is available in $\mathrm{P}$. Opening is the dilation of the erosion of a set $\mathrm{P}$ by a structuring element $\mathrm{Q}$, which can be defined as:

$$
\mathrm{P} \text { o } \mathrm{Q}=(\mathrm{P} \ominus \mathrm{Q}) \oplus \mathrm{Q}
$$

The segmented image is post processed using the Morphological operations so that there is no loss of information.

\subsection{Feature extraction: Integral histogram of gradients, discrete wavelet transform}

Histogram of Gradients (HoG) is a feature descriptor image processing technique used for object detection. Image is decomposed as small squared cells, histogram of gradient is computed in each cells and returns a descriptor for each cell using normalization. The Integral HoG algorithm for the proposed method is shown in Algorithm 2.

Algorithm-2:

Step 1: Divide the image into $8 \mathrm{X} 8$ size

Step 2: Resize the image as 64 X128

Step 3: Calculate the Gradients of $X$ and $Y$ directions in each cell where $G_{x}$ is $X$-Gradient and $G_{y}$ is $Y$-Gradient

Step 4: Calculate the Magnitude and Orientation

$$
\begin{aligned}
& \text { Mag }=\operatorname{sqrt}\left(\left(G_{\mathrm{x}}\right)^{2}+\left(G_{\mathrm{y}}\right)^{2}\right) \\
& \text { Ori }=\tan (\Phi)=\mathrm{G}_{\mathrm{y}} / \mathrm{G}_{\mathrm{x}} \\
& \text { where } \Phi=\tan ^{-1}\left(\mathrm{G}_{\mathrm{y}} / \mathrm{G}_{\mathrm{x}}\right)
\end{aligned}
$$

Step 5: Normalize the Magnitude vector and Orientation Step 6: Obtain descriptor for each cell by Integral Process.

Discrete Wavelet Transform helps to represent the finer variations of signals at various scales [21]. DWT reduce the size of the image without compromising the quality and increases the resolution of the image. Figure 2 shows the subband partitioning of image using DWT.

Algorithm-3:

Step 1: Convert the image into 64 X 64

Step 2: Define the Haar wavelet

Haar function is defined by $\Psi_{\mathrm{n}, \mathrm{k}}(\mathrm{t})=2^{\mathrm{n} / 2} \Psi\left(2^{\mathrm{n}} \mathrm{t}-\mathrm{k}\right), \mathrm{t} \in \mathrm{R}$ 
where $\mathrm{n}, \mathrm{k}$ are integers and $\mathrm{R}$ is real line

Step 3: Apply DWT transform to generate sub-bands LL, LH, HL, HH. [22].

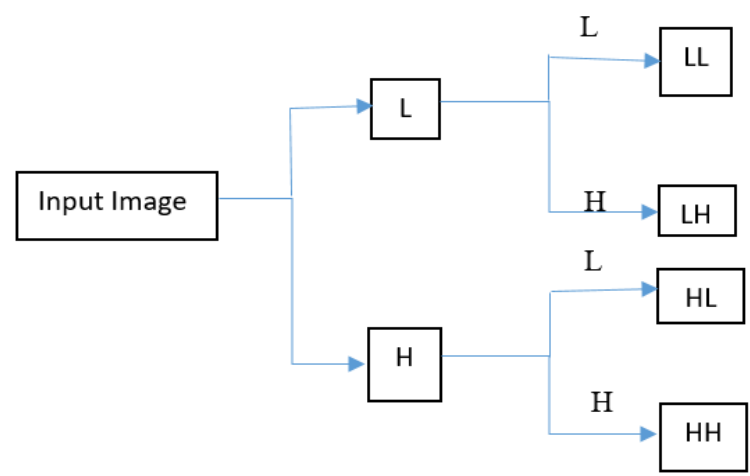

Figure 2. DWT: Sub-band partitioning of image

The combination of Histogram of Gradient (HoG) and Discrete Wavelet Transform (DWT) feature extraction methods helps the researchers to extract the features of teeth, bone and pulp from the Dental Panoramic radiograph. The extracted features are provided as input for the classifier to diagnose the pulpitis.

\subsection{Classification-multi-layer neural network}

Classification of objects into different categories based on feature extraction can be done using pattern recognition. Due to the image limitations and artifacts as discussed in image acquisition, pixel misclassification leads to wrong classification of images [23]. In our proposed work MultiLayer Neural Network (MLNN) is designed to classify the Dental Panoramic Radiographic image as Normal of pulpitis which helps the Dentist in automatic diagnosing of pulpitis.
Multi-Layer Neural Network contains artificial neurons and nodes in more than one layer. It has more interconnected neurons between input and output layer.

\section{Algorithm 4:}

Training:

Step 1: Combine the HoG and DWT feature data into one array tot_array $=\left[\right.$ feat $\left._{\mathrm{dwt}}+\mathrm{feat}_{\mathrm{hog}}\right]$

Step 2: Define the Neural Network Configurations net=feedforward_multilayer net.layer $=3$ net.activation $=$ Relu

Step 3: for $i=1$ : Number of labels for $\mathrm{j}=1$ :Number of feature length train_data $=\{$ feature $\}$ train_labels $=\{$ labels $\}$ end end

Step 4: Train the data

Step 5: Save the model

\section{Testing:}

Step 1: Load the model

Step 2: Load input_image_features

Step 3: Out=simulate(image_feature,model)

Step 4: Get the output label

The features extracted from Histogram of Gradient (HoG) and Discrete Wavelet Transform (DWT) are fed to the MultiLayer Neural Network (MLNN) training model. Based on the trained features, the tested images are classified by the model into two classes as Normal tooth and Pulpitis tooth. Figure 3 shows the block diagram for Pulpitis Classification using Multi-Layer Neural Network from DPR Images. In our proposed research three layers are used in hidden layer which trains the automatic model for better classification.

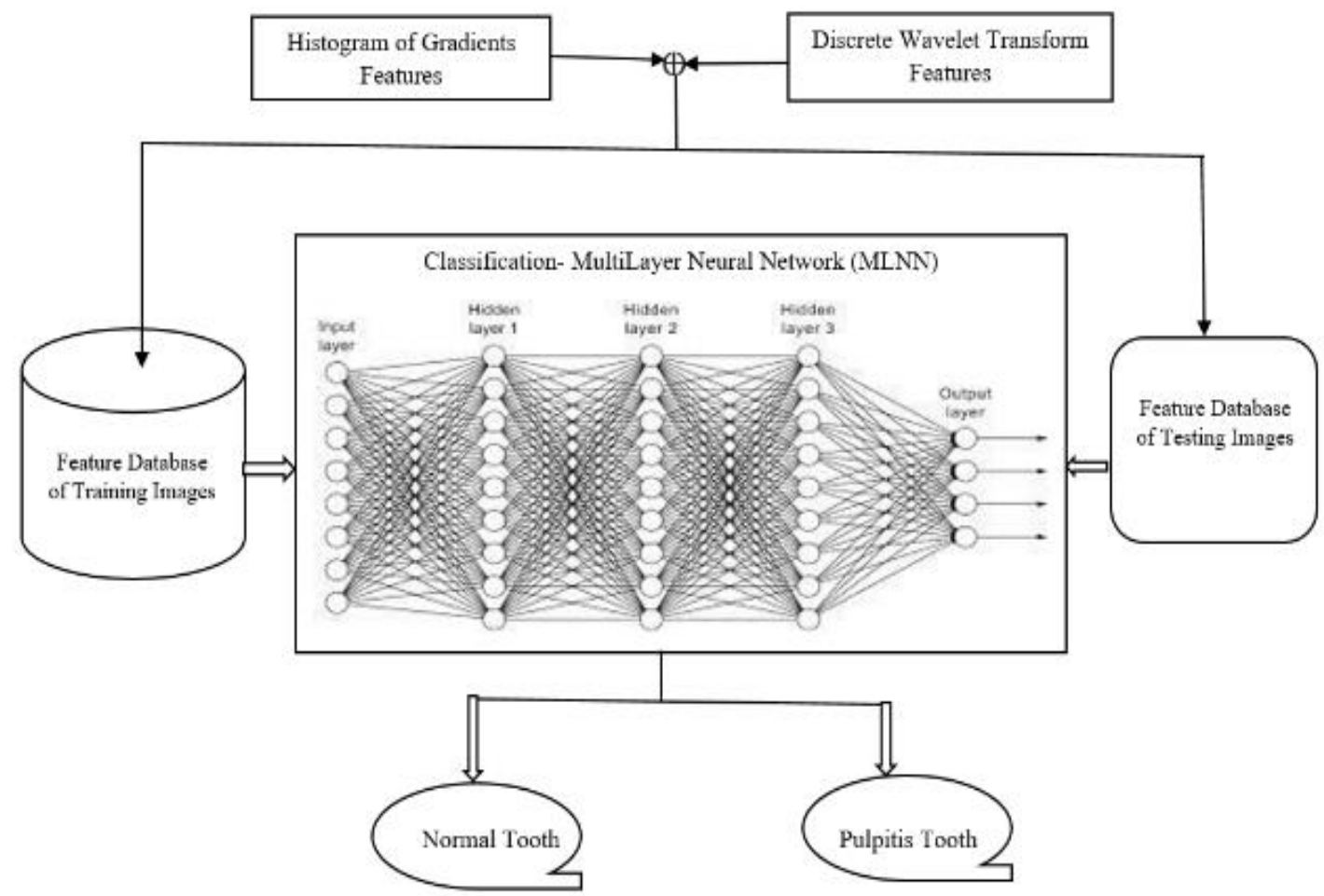

Figure 3. Block diagram for pulpitis classification using multi layer neural network from DPR images 


\section{RESULTS AND DISCUSSION}

The proposed model was developed with a set of 200 Dental Panoramic Radiographic images of subjects aged 30-50 collected from SRM Dental College, Chennai. The size of the images are $1480 \mathrm{X} 2658$ captured with $70 \mathrm{KV}$ to $80 \mathrm{KV}$ voltage and $8 \mathrm{~mA}$ to $10 \mathrm{~mA}$ current. The images are manually grouped and labelled by the Dentist as Normal image and Pulpitis image which helps the researchers to train the automatic model with the traditional Multi-Layer Neural Network Classifier. The obtained results are validated by the dentist and can be used as an assistive tool for their diagnosing and treatment.

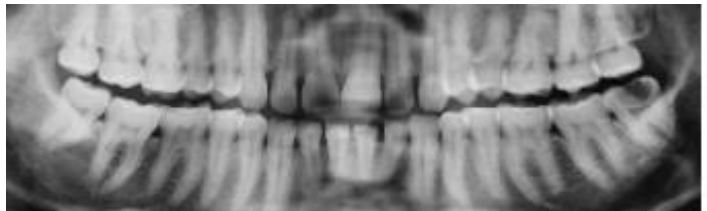

(a) Region of Interest

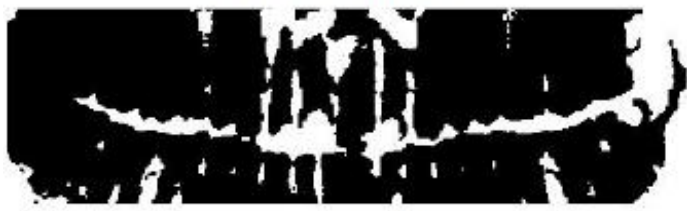

(c) Selected Cluster

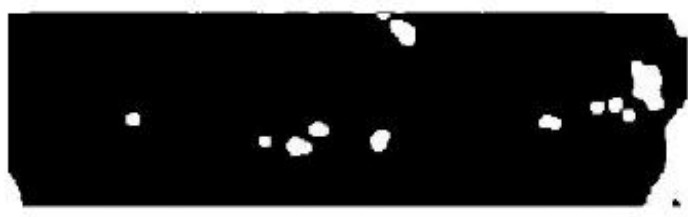

(d) Post- Processing
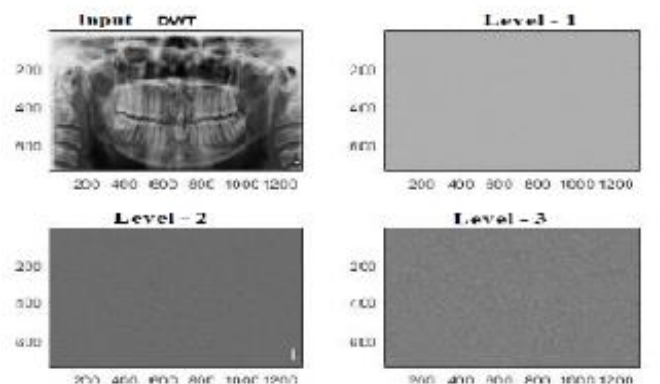

Laval - 3

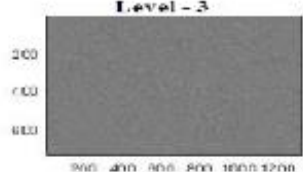

(f) Discrete Wavelet Transform

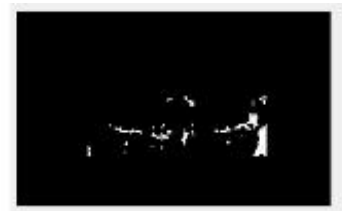

Clnster -3

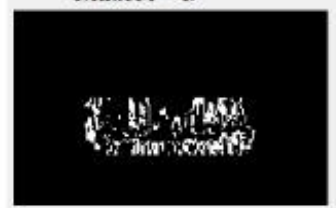

(b) Modified K-Means Cluster

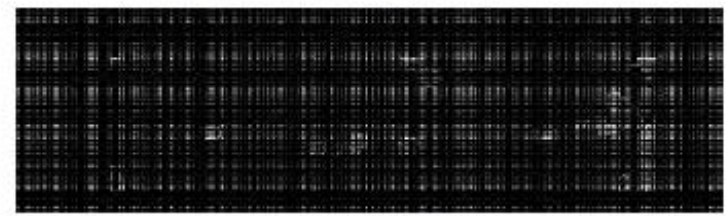

(e) Integral Histogram of Gradients

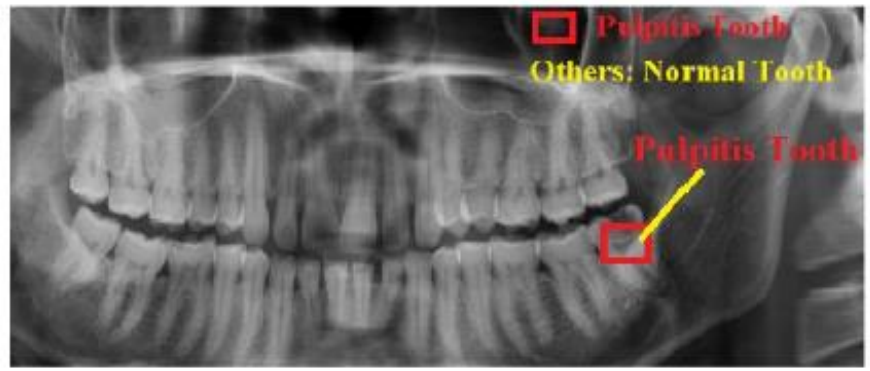

(g) Disgnosis of Pulpitis Tooth

Figure 4. Various stages of implementation using proposed methodologies in diagnosis of pulpitis

Table 1. Confusion matrix outcome parameters - comparison of proposed model with existing methods for 10 sample images

\begin{tabular}{|c|c|c|c|c|c|c|c|c|c|c|c|c|}
\hline \multirow{2}{*}{ Test Images / Stages } & \multicolumn{4}{|c|}{ DWT + NN } & \multicolumn{4}{|c|}{ IHOG + NN } & \multicolumn{4}{|c|}{ DWT+IHOG+MLNN } \\
\hline & TP & FP & FN & TN & TP & FP & FN & $\mathbf{T N}$ & TP & FP & FN & TN \\
\hline Image 1 & 1 & 2 & 2 & 1 & 2 & 1 & 1 & 1 & 3 & 0 & 0 & 1 \\
\hline Image 2 & 3 & 2 & 3 & 0 & 3 & 2 & 3 & 0 & 5 & 0 & 1 & 0 \\
\hline Image 3 & 2 & 2 & 2 & 1 & 3 & 2 & 1 & 1 & 4 & 1 & 0 & 1 \\
\hline Image 4 & 3 & 0 & 3 & 1 & 4 & 2 & 2 & 0 & 5 & 0 & 1 & 0 \\
\hline Image 5 & 4 & 1 & 3 & 1 & 6 & 0 & 1 & 0 & 6 & 1 & 1 & 0 \\
\hline Image 6 & 1 & 2 & 1 & 1 & 2 & 2 & 0 & 1 & 2 & 0 & 0 & 0 \\
\hline Image 7 & 3 & 1 & 1 & 1 & 3 & 1 & 1 & 1 & 3 & 1 & 1 & 0 \\
\hline Image 8 & 2 & 1 & 2 & 1 & 3 & 0 & 1 & 1 & 4 & 0 & 0 & 0 \\
\hline Image 9 & 2 & 2 & 2 & 0 & 3 & 0 & 1 & 0 & 4 & 0 & 0 & 0 \\
\hline Image 10 & 2 & 0 & 2 & 1 & 2 & 0 & 2 & 1 & 4 & 0 & 0 & 0 \\
\hline
\end{tabular}

Table 2. Performance parameters comparison of proposed model (DWT+IHOG+MLNN) with existing methods (DWT+NN) and $(\mathrm{IHOG}+\mathrm{NN})$

\begin{tabular}{ccccc}
\hline Methods & Accuracy & Recall & Precision & F-Score \\
\hline DWT + NN & 64.84 & 69.43 & 68.02 & 68.72 \\
IHOG + NN & 70.53 & 79.13 & 76.17 & 77.62 \\
DWT+IHOG+MLNN & 91.09 & 98.20 & 92.37 & 95.20 \\
\hline
\end{tabular}


Figure 4 shows the output of each stage during the implementation of proposed automatic model. The captured input image is enhanced using Gaussian and Median filter and pre-processed by Histogram Equalization for further enhancement. To facilitate the research work better the Alveolar area of the input image is considered as Region of Interest (Figure 4a). Modified K-means clustering (Figure 4b) is applied to segment the tooth and bony area of Mandibular area and the final cluster is selected (Figure 4c) for further postprocessing (Figure 4d) using morphological operations. The features like tooth, crown, pulp are extracted using Integral Histogram of Gradients (HoG) (Figure 4e) and Discrete Wavelet Transform (DWT) (Figure 4f). The final output of diagnosing pulpitis using the proposed methodologies are shown in Figure 4g with red coloured label and other teeth are considered as Normal teeth. The proposed research work is implemented using the software MATLAB 2020a.

The performance of the proposed method is validated with the Ground Truth (GT) labelled by Dentist and calculated based on the performance measure parameters Accuracy, Sensitivity, Precision and Specificity. The parameters are defined using four terms: True Positive (TP), True Negative (TN), False Positive (FP) and False Negative (FN).

The performance parameters are defined as follows.

$$
\text { Accuracy }=(\mathrm{TP}+\mathrm{TN}) /(\mathrm{TP}+\mathrm{FP}+\mathrm{FN}+\mathrm{TN})
$$

Precision $=\mathrm{TP} /(\mathrm{TP}+\mathrm{FP})$

$$
\text { Recall (or) Sensitivity }=\mathrm{TP} /(\mathrm{TP}+\mathrm{FN})
$$

$$
\text { F-score }=(2 *(\text { Recall } * \text { Precision })) /(\text { Recall }+ \text { Precision })
$$

Table 3. Comparison of confusion matrix parameters with the existing methodologies

\begin{tabular}{ccccc}
\hline Methods & TP & FP & FN & TN \\
\hline DWT + NN & 134 & 63 & 59 & 91 \\
IHOG + NN & 163 & 51 & 43 & 62 \\
DWT+IHOG+MLNN & 218 & 18 & 4 & 7 \\
\hline
\end{tabular}

The research was carried out with DWT+NN and IHoG+NN and the obtained result was not satisfied by the Researchers. The proposed automatic model was developed by combining DWT with IHoG for feature extraction and MultiLayer Neural Network for Classification of Normal tooth and pulpitis tooth and achieved better result than the previous methods.

Table 1 shows the Confusion Matrix Outcome parameters in the Comparison of Proposed Model with Existing Methods for 10 Sample Images. Table 2 and Figure 5 shows the Performance Parameters Comparison of Proposed Model $(\mathrm{DWT}+\mathrm{IHOG}+\mathrm{MLNN})$ with Existing Methods (DWT+NN) and (IHOG+NN) with Accuracy 91.09\%, Recall 98.20\%, Precision 92.37\% and F-score 95.20\%. Table 3 and Figure 6 shows the results of Confusion Matrix of proposed automatic assistive model with 218 True Positive values which is better compared to other methods.

In a similar work by Tumbelaka et al., pulpitis was identified from Periapical Radiograph using the Gaussian filter, texture descriptors and Artificial Neural Network analysis. This method diagnosed the pulp indications as normal, pulpitis and death tooth but the authors felt that the radiography reading has to be digitized to get better diagnosis validation
[24]. The proposed method achieved the accuracy of $91.09 \%$ which is better compared to the previous methodologies and can be used as an assistive tool by the Dentist in diagnosing and treatment of Pulpitis from Dental Panoramic Radiograph.

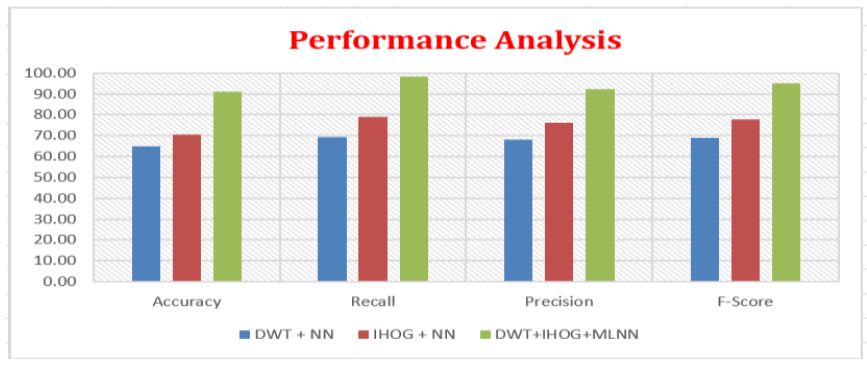

Figure 5. Performance parameters of classification accuracy, recall, precision, \& F-score

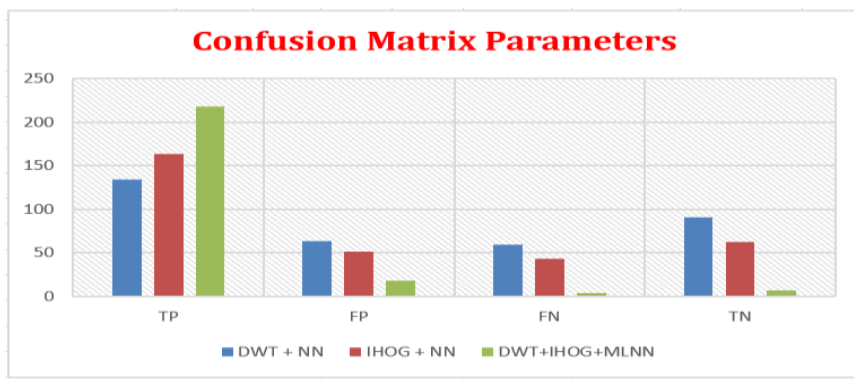

Figure 6. Confusion matrix parameters comparison of proposed model with existing methods

\section{CONCLUSION}

The research work proposes an automatic model to diagnose pulpitis from Dental panoramic Radiography. Gaussian and Median filters are used for noise removal and Histogram Equalization helps in enhancement of the captured image. Modified K-means Clustering helps the researchers to segment the teeth, pulp and bony area of the alveolar area. Integral Histogram of Gradients combined with Discrete Wavelet Transform extracts the features for classification process. Multi-Level Neural Network (MLNN) classifier along with proposed Segmentation and Feature extraction methods achieves the better accuracy of $91.09 \%$, Recall of $98.20 \%$, Precision of $92.37 \%$ and F-score of $95.20 \%$ which is better compared to the previous methodologies. Our proposed model can be used as an assistive tool by the Dentist in diagnosing Pulpitis which reduces their diagnostic effort and workload.

\section{REFERENCES}

[1] Wang, C.W., Huang, C.T., Lee, J.H., Li, C.H., Chang, S.W., Siao, M.J., Lai, T.M., Ibragimov, B., Vrtovec, T., Ronneberger, O., Fischer, P., Cootes, T.F., Lindner, C. (2016). A benchmark for comparison of dental radiography analysis algorithms. Medical Image Analysis, 31: 63-76. https://doi.org/10.1016/j.media.2016.02.004

[2] Kumar, R., Khamdete, N., Priya, E. (2011). Extraoral periapical radiography: An alternative approach to intraoral periapical radiography. Imaging Science in Dentistry, 41(4):

161-165. 
https://doi.org/10.5624/isd.2011.41.4.161

[3] Shah, N. (2018). Oral and dental diseases: Causes, prevention and treatment strategies. NCMH Background Papers- Burden of Disease in India, Division of Conservative Dentistry and Endodontics, Chapter, pp. 275-298.

[4] Ali, S.G., Mulay, S. (2015). Pulpitis: A review. IOSR Journal of Dental and Medical Sciences, 14(8): 92-97. https://doi.org/10.9790/0853-14869297

[5] Rechenberg, D.K., Galicia, J.C., Peters, O.A. (2016). Biological markers for pulpal inflammation: A systematic review. PLoS One, 11(11): 1-24. https://doi.org/10.1371/journal.pone.0167289

[6] Glickman, G.N., Schweitzer, J.L. (2013). Endodontics: Colleagues for excellence. Published for the Professional Community by the American Association of Endodontists.

[7] Talabani, R.M., Ali, A.J., Abdulkareem, B.N. (2016). Clinical and radiographic evaluation of pulpal and periradicular tissue. International Journal of Pharmaceutical Sciences and Research, 7(12): 50725076. https://doi.org/10.13040/IJPSR.09758232.7(12).5072-76

[8] Abella, F., Patel, S., Duran-Sindreu, F., Mercadé, M., Bueno, R., Roig, M. (2012). Evaluating the periapical status of teeth with irreversible pulpitis by using conebeam computed tomography scanning and periapical radiographs. Journal of Endodontics, 38(12): 1588-1591. https://doi.org/10.1016/j.joen.2012.09.003

[9] Saidi, A., Naaman, A., Zogheib, C.(2015). Accuracy of cone-beam computed tomography and periapical radiography in endodontically treated teeth evaluation: A five-year retrospective study. Journal of International Oral Health, 7(3): 15-19.

[10] Chattopadhyay, S., Davis, R.M., Menezes, D.D., Singh, G., Acharya, R.U., Tamura, T. (2012). Application of Bayesian classifier for the diagnosis of dental pain. Journal of Medical Systems, 36: 1425-1439. https://doi.org/10.1007/s10916-010-9604-y

[11] Zanine, M., Meyer, E., Simon, S. (2017). Pulp inflammation diagnosis from clinical to inflammatory mediators: A systematic review. Journal of Endodontics, 43(7): https://doi.org/10.1016/j.joen.2017.02.009

[12] Shafiei, F., Fekri-Ershad, S. (2020). Detection of lung cancer tumor in CT scan images using novel combination of super pixel and active contour algorithms. Traitement du Signal, 37(6): 1029-1035. https://doi.org/10.18280/ts.370615

[13] Croock, M.S., Khudhur, S.D., Taqi, A.K. (2016). Edge detection and features extraction for dental X-ray. Engineering and Technology Journal, 34(13): 2420-2432.

[14] Bendjillali, R.I., Beldgham, M., Merit, K., Taleb-Ahmed, A. (2019). Improved facial expression recognition based on DWT feature for deep CNN. Electronics, 8(3): 1-16. https://doi.org/10.3390/electronics8030324

[15] Halder, A., Chatterjee, S., Dey, D., Kole, S., Munshi, S. (2020). An adaptive morphology based segmentation technique for lung nodule detection in thoracic CT image. Computer Methods and Programs in Biomedicine, 197: 105720. https://doi.org/10.1016/j.cmpb.2020.105720

[16] Lakhani, K., Minocha, B., Gugnani, N. (2016). Analyzing edge detection techniques for feature extraction in dental radiographs. Recent Trends in Engineering and Material Sciences, 8: 395-398. https://doi.org/10.1016/j.pisc.2016.04.087

[17] Yadav, A.K., Roy, R., Jumar, R., Kumar, C.S., Kumar, A.P. (2015). Algorithm for de-noising of color images based on median filter. 2015 Third International Conference on Image Information Processing (ICIIP), pp. 428-432. https://doi.org/10.1109/ICIIP.2015.7414811

[18] Kathirvelu, D., Vinupritha, P., Kalpana, V. (2019). A computer aided diagnosis system for measurement of mandibular cortical thickness on dental panoramic radiographs in prediction of women with low bone mineral density. Journal of Medical Systems, 43: 148. https://doi.org/10.1007/s10916-019-1268-7

[19] Rad, A.E., Shafry, M., Rahim, M.S.M., Norouzi, A. (2013). Digital dental X-ray image segmentation and feature extraction. TELKOMNIKA Indonesian Journal of Electrical Engineering, 11(6): 3109-3114.

[20] Bharathi, B.S., Swamy, K.V. (2020). Effective image segmentation using modifies K-means technique. 2020 4th International Conference on Trends in Electronics and Informatics (ICOEI)(48184), pp. 757-762. https://doi.org/10.1109/ICOEI48184.2020.9142910

[21] Georgieva, V., Petrov, P., Zlatareva, D. (2021). Medical image processing based on multidimensional wavelet transforms - advantages and trends. AIP Conference Proceedings, $\quad 2333$ : 020001. https://doi.org/10.1063/5.0041869

[22] Ammah, P.N.T., Owusu, E. (2019). Robust medical image compression based on wavelet transform and vector quantization, Informatics in Medicine Unlocked, 15: 100183. https://doi.org/10.1016/j.imu.2019.100183

[23] Chen, Q., Zhao, Y., Liu, Y., Sun, Y., Yang, C., Li, P., Zhang, L., Gao, C. (2021). MSLPNet: Multi-scale location perception network for dental panoramic $\mathrm{x}$-ray image segmentation, Neural Computing and Applications, 33: 10277-10291. https://doi.org/10.1007/s00521-021-05790-5

[24] Tumbelaka, B.Y., Oscandar, F., Baihaki, F.N., Sitam, S., Rukmo, M. (2014). Identification of pulpitis at dental Xray periapical radiography based on edge detection, texture description and artificial neural networks. Saudi Endodontic Journal, 4(3): 115-121. https://doi.org/10.4103/1658-5984.138139 